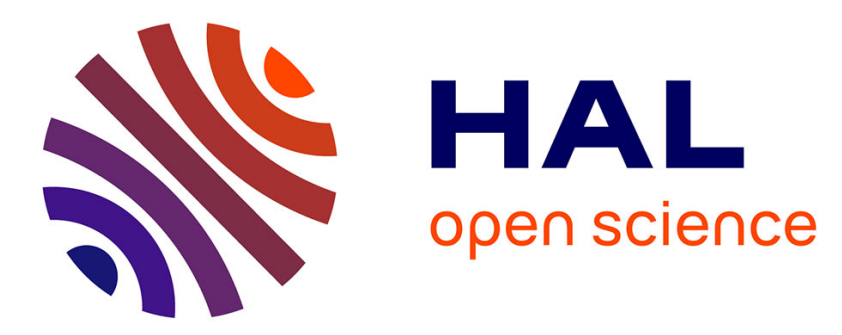

\title{
Performance of an Adaptive Notch Filter for Spectral Analysis of Coherent Lidar Signals
}

Jean-Luc Zarader, Gérard Ancellet, Alain Dabas, Nacer K. M'Sirdi, Pierre H. Flamant

\section{- To cite this version: \\ Jean-Luc Zarader, Gérard Ancellet, Alain Dabas, Nacer K. M'Sirdi, Pierre H. Flamant. Performance of an Adaptive Notch Filter for Spectral Analysis of Coherent Lidar Signals. Journal of Atmospheric and Oceanic Technology, 1996, 13 (1), pp.16-28. insu-02902272}

\section{HAL Id: insu-02902272 \\ https://hal-insu.archives-ouvertes.fr/insu-02902272}

Submitted on 18 Jul 2020

HAL is a multi-disciplinary open access archive for the deposit and dissemination of scientific research documents, whether they are published or not. The documents may come from teaching and research institutions in France or abroad, or from public or private research centers.
L'archive ouverte pluridisciplinaire HAL, est destinée au dépôt et à la diffusion de documents scientifiques de niveau recherche, publiés ou non, émanant des établissements d'enseignement et de recherche français ou étrangers, des laboratoires publics ou privés. 


\title{
Performance of an Adaptive Notch Filter for Spectral Analysis of Coherent Lidar Signals
}

\author{
JEAN-LUC ZARADER \\ Perception, Automatique et Reseaux Connexionnistes, Paris, France \\ GÉrARd ANCELLET \\ Service d'Aéronomie du CNRS, Paris, France \\ Alain Dabas \\ Laboratoire de Météorologie Dynamique, Ecole Polytechnique, Paliseau, France \\ NACER K. M'SIRDI \\ Laboratoire de Robotique de Paris, Paris, France \\ PierRe H. Flamant \\ Laboratoire de Météorologie Dynamique, École Polytechnique, Palasieau, France \\ (Manuscript received 22 July 1992, in final form 4 January 1994)
}

\begin{abstract}
An adaptive notch filter (ANF) is proposed for range-resolved frequency estimates of Doppler lidar atmospheric returns. The ANF is based on the spectral filtering of lidar return to remove the atmospheric contribution from noise. An adaptive algorithm is used to retrieve the filter parameters at a time $k$ knowing both the input signal and filter output at times $k-i$, where $i=[1, k]$. It is shown that ANF performs well at low SNR $(-5$ $\mathrm{dB}$ ) compared to the poly-pulse-pair (PPP) estimator currently used for Doppler lidar signal processing. The standard deviation of frequency estimates is $0.01 F_{S}-0.02 F_{S}\left(F_{S}\right.$ is the sampling frequency) at SNR $=-5 \mathrm{~dB}$, depending on the signal spectral width. It corresponds to a wind velocity uncertainty of $2-4 \mathrm{~m} \mathrm{~s}^{-1}$ for $F_{s}=40$ $\mathrm{MHz}$ and a laser wavelength $\lambda=10 \mu \mathrm{m}$. The ANF also proved to perform better than PPP in tracking a timevarying frequency, and in the presence of a colored noise.
\end{abstract}

\section{Introduction}

Pulsed coherent Doppler lidar is a powerful technique to document the three-dimensional wind field in clear-air condition (Menzies and Hardesty 1989). Ground-based systems have been operated by NOAA WPL (Post and Neff 1986; Neiman et al. 1988) and NASA MSFC (Rothermel et al. 1985) since the late 1970 s, and airborne operation has been demonstrated in the meantime (Bilbro et al. 1984, 1986). During the past decade, a considerable effort was devoted to an improvement of the $10-\mu \mathrm{m}$ technology currently used in Doppler lidar, with a special emphasis put on frequency stability of high-energy $\mathrm{CO}_{2}$ lasers (Hardesty et al. 1983; Dolfi-Bouteyre et al. 1988). Signal process-

\footnotetext{
Corresponding author address: Jean-Luc Zarader, Perception, Automatique et Reseaux Connexionnistes, Universite Pierre et Marie Curie, 4 Place Jussieu, 75252 Paris, Cedex 05, France.
}

ing, which is another issue, benefited greatly from algorithm development conducted in the weather radar domain, like the pulse-pair frequency estimator (Doviak and Zrnic 1984), for example. These estimators are compliant with a rather stringent requirement in Doppler lidar in which the signals have to be processed on a single-shot basis. Only an accumulation of lidar spectra afterward is relevant to decrease the variance. In addition, the experimenter faces a characteristic of pulsed coherent Doppler lidar that is a low pulse repetition frequency of a few tens to hundreds Hertz for high-energy laser transmitters. Another limitation is brought by signal decorrelation due to inhomogeneities in the scattering volume (such as sharp gradient in backscatter, velocity turbulence, wind shear) or instrumental factor like a frequency chirp of the transmitter emission. All these phenomena result in decorrelation times shorter than pulse duration (Ancellet et al. 1989) and consequently signal spectra broader than pulselimited spectra. Accordingly, the spectral width can be 
of the order of, or even greater than, the corresponding required accuracy. Considering these limitations, the main improvement foreseen to expand the field of lidar application is to achieve better frequency estimator performance (i.e., smallest frequency error) at low signalto-noise ratio (SNR) and high spatial resolution. This improvement will benefit the mature $10-\mu \mathrm{m}$ technology, as well as the $1-\mu \mathrm{m}$ technology and emerging $2-\mu \mathrm{m}$ technology.

For many years now, coherent lidar signal processing resorted to pulse-pair (PP) and poly-pulse-pair (PPP) frequency estimators (Miller and Rochwarger 1972; Zrnić 1979; Lee and Lee 1980). They are easy to implement, and their accuracy is fairly good at moderate SNR. As a result, most coherent lidars in operation use PP and PPP estimators. However, the recent availability of fast-floating point processors questions this widely accepted statement, since these processors enable the implementation of new algorithms (demanding more computer power) that could perform better at low SNR. Regarding the applications, it turns out they could be more appropriate to Doppler lidar processing.

The purpose of the present paper is to discuss the performance of the adaptive linear filtering technique applied to Doppler lidar processing, and adaptive notch filter (ANF hereafter) in particular. The capability of ANF to improve Doppler lidar signal processing is addressed in section 4 , where, according to the current status in Doppler lidar signal processing, a comparison of ANF performance with respect to PPP is conducted. Beforehand, we present briefly the linear filtering technique in section 2, and then the ANF algorithm is discussed in section 3 .

\section{Linear filtering technique}

The linear filtering technique consists in defining a linear filter, a so-called whitening filter, that is able to remove the atmospheric return bearing the wind field information from detection noise. The Doppler frequency is then related to the whitening filter parameters. The linear filtering algorithms are all based on an assumption made on the capability to model the lidar signal at detection level, at least in good approximation, by the output of a linear filter when a white noise is used as an input signal. The linear filter is a so-called predictor filter. The predictor is the inverse of a whitening filter.

The modern linear filtering techniques appeared during the late 1960s. The maximum entropy method for instance (Burg 1967), which was first proposed for geophysical data processing, is in fact a filtering technique where the predictor filter is all-pole or auto-regressive.

Regarding the adaptive versions of the linear filtering technique, the whitening filter parameters are assumed to vary slowly in time according to the quasi stationarity of the lidar signal. Adaptive estimators then consist in correcting the parameter filters from one time sample to the next instead of recomputing them at each step independently of the previous estimates. Two main procedures for correction exists: the gradient method (Widrow and Stearn 1985), or recursive least squares method (Ljung and Soderström 1983) based on the matrix inversion lemma. The adaptive notch filter, proposed by Nehoraï (1985) according to Ljung's works, belongs to the second category.

\section{Adaptive notch filter (ANF)}

In ANF technique, the whitening filter is assumed to be a notch filter. The notch filter is an ARMA of order $2 \times 2$ (which corresponds to two zeros and two poles) where the poles and zeros are constrained. The relationship between the notch filter and signal frequency is then simple and leads to a fast adaptive estimator.

\section{a. Notch filter}

The notch filter is designed to remove one frequency component of a sampled signal without changing the others. To do so, a first attempt is to use a finite-impulse response (FIR) filter $H$ response $H(z)$ is

$$
H(z)=\left(1-z_{1} z^{-1}\right)\left(1-z_{2} z^{-2}\right),
$$

where $Z_{1}$ and $Z_{2}$ are the filter zeros:

$$
\begin{aligned}
& z_{1}=\exp \left(2 i \pi f_{d} T_{s}\right) \\
& z_{2}=z_{1}^{*}=\exp \left(-2 i \pi f_{d} T_{s}\right),
\end{aligned}
$$

where $f_{d}$ designates the Doppler spectral component to be removed, and $T_{s}$ denotes the sampling time. Figure 1 shows the transfer function $|H(f)|$ for such a filter,

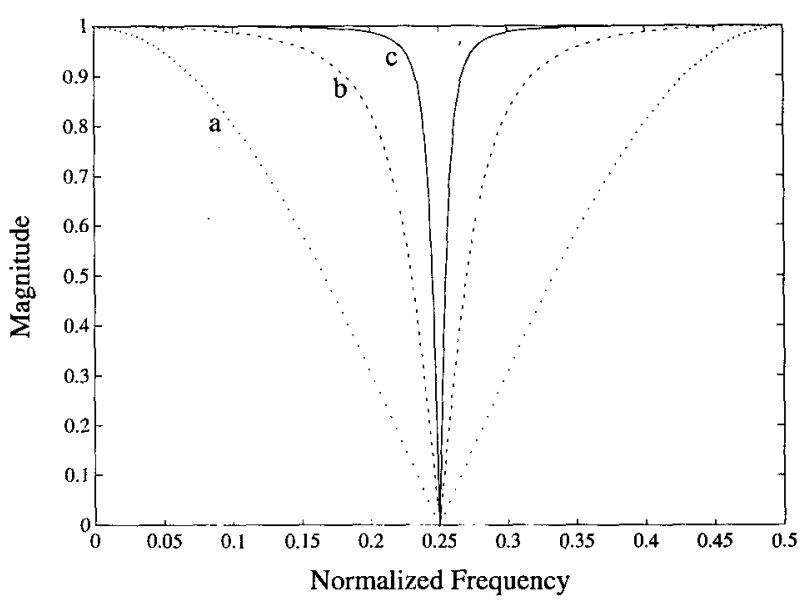

FiG. 1. Filter transfer functions of (a) the moving-average filter, (b) the notch filter with $r=0.8$, and (c) the notch filter with $r=0.95$. All filters are centered about $0.25 F_{s}$. 


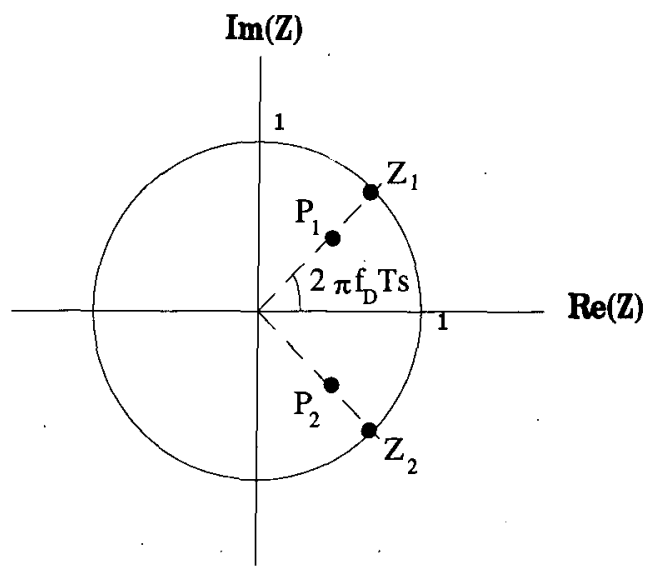

FIG. 2. Notch filter zeros and poles in the $z$ complex plane.

which turns out to be so large that other spectral components are likely to be altered. To improve the selectivity of the notch filter (Glover 1977), one can introduce two poles $p_{1}$ and $p_{2}$, with the same phase as $z_{1}$ and $z_{2}$ (Fig. 2) and close to them in the complex plane:

$$
\frac{p_{1}=r z_{1}}{p_{2}=r z_{2}}
$$

where $0<r<1$. It leads to the notch filter transfer function:

$$
\begin{aligned}
H(z)= & \frac{\left(1-z_{1} z^{-1}\right)\left(1-z_{2} z^{-1}\right)}{\left(1-p_{1} z^{-1}\right)\left(1-p_{2} z^{-1}\right)} \\
& =\frac{1-2 \cos \left(2 \pi f_{d} T_{s}\right) z^{-1}+z^{-2}}{1-2 r \cos \left(2 \pi f_{d} T_{s}\right) z^{-1}+r^{2} z^{-2}} .
\end{aligned}
$$

The poles $p_{1}$ and $p_{2}$ remain within the unit circle of the $z$ plane to ensure the stability of the notch filter. Figure 1 displays the transfer functions for $r=0.80$ and 0.95 . We notice that $r$ tunes the filter bandwidth since $B$ $=1-r$. Denoting $x(k)$ the input signal at time $k$ and $s(k)$ the output of the filter, the recurrence relationship between $x(k)$ and $s(k)$ corresponding to (4) is (Rabiner and Gold 1975)

$$
\begin{aligned}
x(k)=-a_{1} x(k-1)-x(k-2)+s(k) & \\
& +a_{1} r s(k-1)+r^{2} s(k-2),
\end{aligned}
$$

where the filter parameter $a_{1}$ is related to the filter central frequency:

$$
a_{1}=-2 \cos \left(2 \pi f_{d} T_{s}\right) .
$$

An estimate of the signal frequency using the notch filter is made as follows. Let us consider for instance that the signal is made of a sine wave $x(k)=A$ $\cos \left(2 \pi f_{d} k T_{s}+\varphi\right)$ where the phase $\varphi$ is unknown, plus a zero-mean white noise $n(k)$ of power $\sigma_{n}^{2}$. When filtered by the notch filter centered about $f_{d}$, the sine wave is removed so the output $s(k)$ can be considered as an estimate of the noise contribution $n(k)$ (Fig. 3) and will therefore be denoted $\tilde{n}(k)$ :

$$
\begin{aligned}
\tilde{n}(k)=x(k) & +\hat{a}_{1}(k) x(k-1)+x(k-2) \\
& -\hat{a}_{1}(k) r \tilde{n}(k-1)-r^{2} \tilde{n}(k-2),
\end{aligned}
$$

where $\hat{a}_{1}(k)$ is the estimate of parameter $a_{1}$ at time $k$. Let us denote

$$
\begin{aligned}
p(k)=-\hat{a}_{1}(k) x(k-1)-x(k-2) & \\
& +\hat{a}_{1}(k) r \tilde{n}(k-1)+r^{2} \tilde{n}(k-2) .
\end{aligned}
$$

We then have

$$
\tilde{n}(k)=x(k)-p(k)
$$

and it can be shown (see appendix A) that $p(k)$ is the optimal predictor of $x(k)$ in a least squares sense since it minimizes the mean quadratic error $J_{k}=E\left[\tilde{n}^{2}(k)\right]$, where $E$ denotes the mathematical expectation.

As a consequence, the ANF works as follows. At time $k$, the quadratic error $J_{k}$ is first minimized with respect to the parameter $a_{1}$ leading to the estimate $\hat{a}_{1}(k)$, in order to minimize the prediction error. The optimal predictor $p(k)$ is then calculated with $(8)$, and
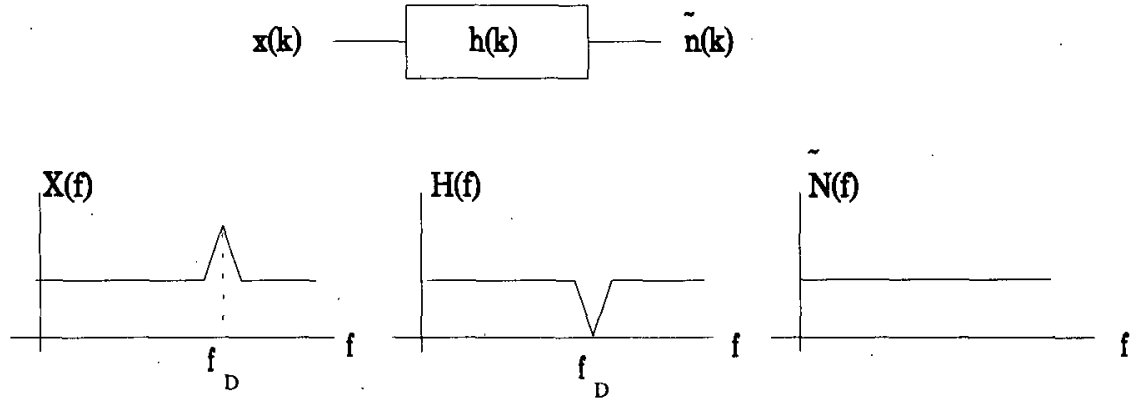

FIG. 3. The notch filter centered about $f_{d}$ removes the useful signal at frequency $f_{d}$ from the input signal $x(k)$ leaving only the noise contribution. 
then the prediction error $\tilde{n}(k)$ with $(9)$. The ANF estimation can then go on to the next step $k+1$.

\section{b. Parametric adaptive algorithm}

As shown in section 3a, the ANF estimator needs to minimize the quadratic error $J_{k}$ with respect to the filter parameter $a_{1}$. This minimization is the critical point of the method for it is generally computer time consuming. Two adaptive methods converging toward a minimum of a so-called cost function can however be used to simplify the procedure: the gradient method and the recursive least squares method due to Ljung and Soderström (1983). In the present work, we used a recursive maximum likelihood (RML) algorithm, which, according to Nehorai (1985), is better suited to a notch filter.

Let us define $\phi(k)$ the transposed vector related to the input/output of the filter, and $\theta(k)$ the transposed vector of the filter parameter:

$$
\begin{aligned}
\boldsymbol{\phi}(k)=[-x(k-1)+ & r \tilde{n}(k-1), \\
& \left.-x(k-2)+r^{2} \tilde{n}(k-2)\right]^{\mathrm{T}} \\
\boldsymbol{\theta}(k)= & {\left[\hat{a}_{1}(k), 1\right]^{\mathrm{T}}, \quad(10) }
\end{aligned}
$$

where $\mathrm{T}$ denotes the transpose vector. The optimal predictor is then [cf. (8)]

$$
p(k)=\boldsymbol{\theta}^{\mathrm{T}}(k) \boldsymbol{\phi}(k) .
$$

The quadratic error $J_{k}$ at time $k$ is then equal to

$$
J_{k}=E\left\{\left[x(k)-\boldsymbol{\theta}^{\mathrm{T}}(k) \phi(k)\right]^{2}\right\} .
$$

It cannot be calculated unless the statistical properties of the signal are known a priori, which is generally not the case. Therefore an estimate is made through the recursive procedure:

$$
\hat{J}_{k}=\frac{1}{k} \sum_{i=1}^{k} \lambda_{k}(i)\left[x(i)-\boldsymbol{\theta}^{\mathrm{T}}(k) \boldsymbol{\phi}(i)\right]^{2},
$$

where

$$
\lambda_{k}(i)=\lambda_{0}^{k-i} .
$$

The parameter $\lambda_{0}$ defines a weighting function for the contribution of the previous time steps to the quadratic error $\hat{J}_{k}$. It is generally chosen within the interval [0.8, 1]. As a matter of fact, the parameter $\lambda_{0}$ tunes the time resolution of ANF estimates. When $\lambda_{0}=1$, for instance, the estimate is performed on the whole signal from the beginning to step $k$. On the contrary, when $\lambda_{0}$ $=0.8$, the weight given to component $k-31$ is less than $10^{-3}\left(\lambda_{0}^{31} \leqslant 10^{-3}\right)$, and thus negligible. Through $\lambda_{0}$, the time window corresponding to an ANF estimate is fixed. For the weighting function is a negative exponential, there exists no straightforward definition for the time resolution. In this paper, we set the time res- olution of an estimate as the time delay $m T_{s}$ when the weight $\lambda_{0}^{m}$ becomes lower than $e^{-1}: m=\left[\log \left(\lambda_{0}\right)\right]^{-1}$. For instance, when $\lambda_{0}=0.8$, the time resolution is equal to 5 ; when $\lambda_{0}=0.9$ and 0.95 , it is equal to 10 and 20 , respectively.

The estimation $\hat{J}_{k}$ defined by (13) of the quadratic error $J_{k}$ has a great advantage. As it is shown in appendix $\mathrm{B}$, its recurrent form leads to a recursive relationship on $\boldsymbol{\theta}(k)$ :

$$
\boldsymbol{\theta}(k)=\boldsymbol{\theta}(k-1)+F(k-1) \Psi(k) \epsilon(k),
$$

where $\Psi(k)$ is recursively calculated:

$\Psi(k)=\boldsymbol{\phi}(k)-\hat{a}_{1}(k-1) r \Psi(k-1)-r^{2} \Psi(k-2)$,

and where $F(k-1)$ and $\epsilon(k)$ are defined by

$$
\begin{aligned}
& F(k-1)=\left[\sum_{i=1}^{k-1} \lambda_{0}^{k-1-i} \Psi(i) \phi^{\mathrm{T}}(i)\right]^{-1} \\
& \epsilon(k)=\frac{x(k)-\boldsymbol{\phi}^{\mathrm{T}}(k) \boldsymbol{\theta}(k-1)}{\lambda_{0}+\boldsymbol{\phi}^{\mathrm{T}}(k) F(k-1) \Psi(k)} .
\end{aligned}
$$

The whole ANF procedure is as follows. After some initialization of $\Psi(0), \Psi(1), \tilde{n}(0), \tilde{n}(1), \theta(0)$, and $\boldsymbol{\theta}(1)$, and beyond the time step $2, \boldsymbol{\phi}(k), \Psi(k), F(k$ $-1)$, and $\epsilon(k)$ are calculated from (10), (16), and (17), respectively. The filter parameter vector $\boldsymbol{\theta}(k)$ is then recursively calculated from (15). Once the new filter parameter is estimated, the prediction error $\tilde{n}(k)$ is retrieved using (11) and (9). The procedure can then go on to the next time step.

As it is adaptive, the convergence of ANF from initial values toward the true estimates, or when some nonstationarity occurs during the signal, is of prime importance. The time needed for convergence depends on the quality of the initialization (are the signal characteristics close or far from the initial values), or the degree of the nonstationarity (are the previous signal characteristics close or far from the new values), but also on parameters $\lambda_{0}$ and $r$. We remind the readers that $\lambda_{0}$ sets the time resolution of the estimate, and $r$ the notch filter bandwidth. A faster convergence is obtained when $\lambda_{0}$ and $r$ are small. The time resolution is then better, enabling the ANF to catch the changing signal characteristics faster, and the notch filter selectivity is relaxed, enabling the ANF to catch the signal frequency more easily. But at the same time, it leads to a greater variance of estimates once the convergence is ensured, since now the estimator is more sensitive to random fluctuation (speckle and noise). It is the reason why both $\lambda_{0}$ and $r$ are tuned as the estimation procedure goes on. As an example, in the simulations we conducted in the frame of this study, we tuned $\lambda_{0}$ from 0.8 initially to 0.95 , and $r$ from 0.8 to 0.95 .

The constraint imposed to the zeros and poles of the notch filter makes the convergence of the estimator 

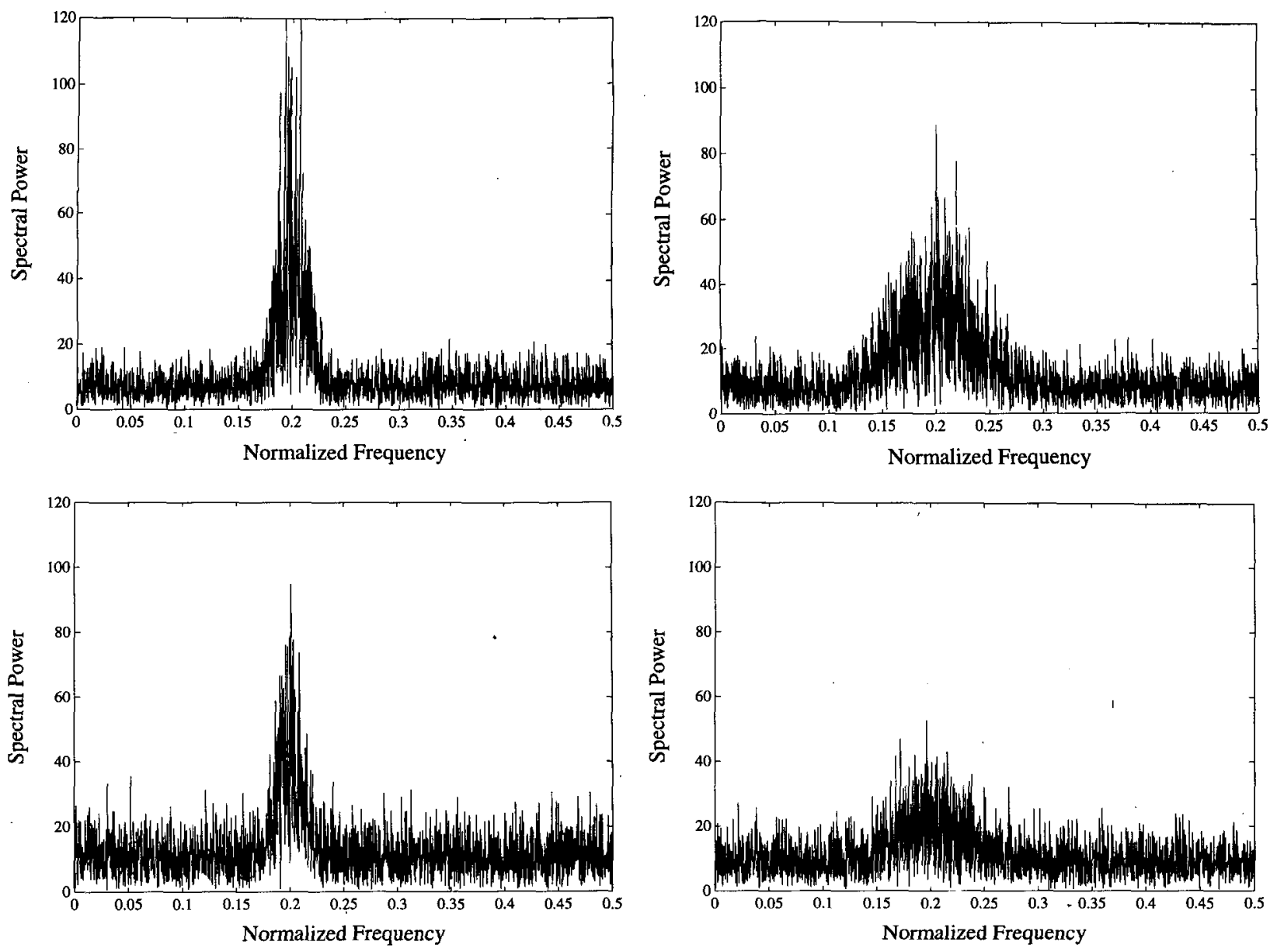

FIG. 4. Examples of simulated power spectra with various SNR and spectral widths $\sigma_{d}$ : (a) $\mathrm{SNR}=0 \mathrm{~dB}$ and $\sigma_{d^{\prime}}=0.01 F_{s,}$ (b) $0 \mathrm{~dB}, 0.03 F_{s}$, (c) $-5 \mathrm{~dB}, 0.01 F_{s}$, and (d) $-5 \mathrm{~dB}, 0.03 F_{s}$.

faster than with a general ARMA model. The ARMA filter has, however, more degrees of freedom and can thus be better adapted to the signal features. For instance, it is better adapted to an analysis of the spectral width (Palmer et al. 1991). The adequacy between the filter model inferring the estimator and the signal leads to a better accuracy. In particular, the CramerRao lower bound for frequency estimation is asymptotically reached when the signal fits the filter model (Ljung and Soderström 1983). As far as the Doppler lidar is concerned, the notch filter model is only an approximate.

\section{Numerical simulations and performance}

The performance of ANF can be evaluated using the following two different approaches:

1) a calculation of the bias and standard deviation of the estimates on stationary signals and once the convergence is reached; and
2) a full spectral analysis of nonstationary signals with a time-varying frequency for instance.

We considered a $20-\mathrm{MHz}$ bandwidth and used a sampling frequency $F_{s}=40 \mathrm{MHz}$. These figures correspond to a velocity range of $\pm 50 \mathrm{~m} \mathrm{~s}^{-1}$ for a $\mathrm{CO}_{2}$ lidar working at $10 \mu \mathrm{m}$. The ANF estimates are systematically compared to the PPP estimates using four lags [ since it is recognized as one of the most efficient complex covariance estimator for SNR and spectral width (Mahapatra and Zrnić 1983) encountered in lidar signals ]. The PPP version we considered is due to Zrnić and Mahapatra (1983) and referenced as PPPPO in their paper. Another complex covariance PPP estimator was indeed proposed by Lee and Lee in 1978. Because of the lack of scientific literature on Lee's estimator, together with a first study we conducted that showed the improvement is rather weak (at $\mathrm{SNR}=-5 \mathrm{~dB}$ and for a spectral width of $0.01 F_{s}$, the standard deviation is $4 \%$ of $F_{s}$ with Lee's PPP and 

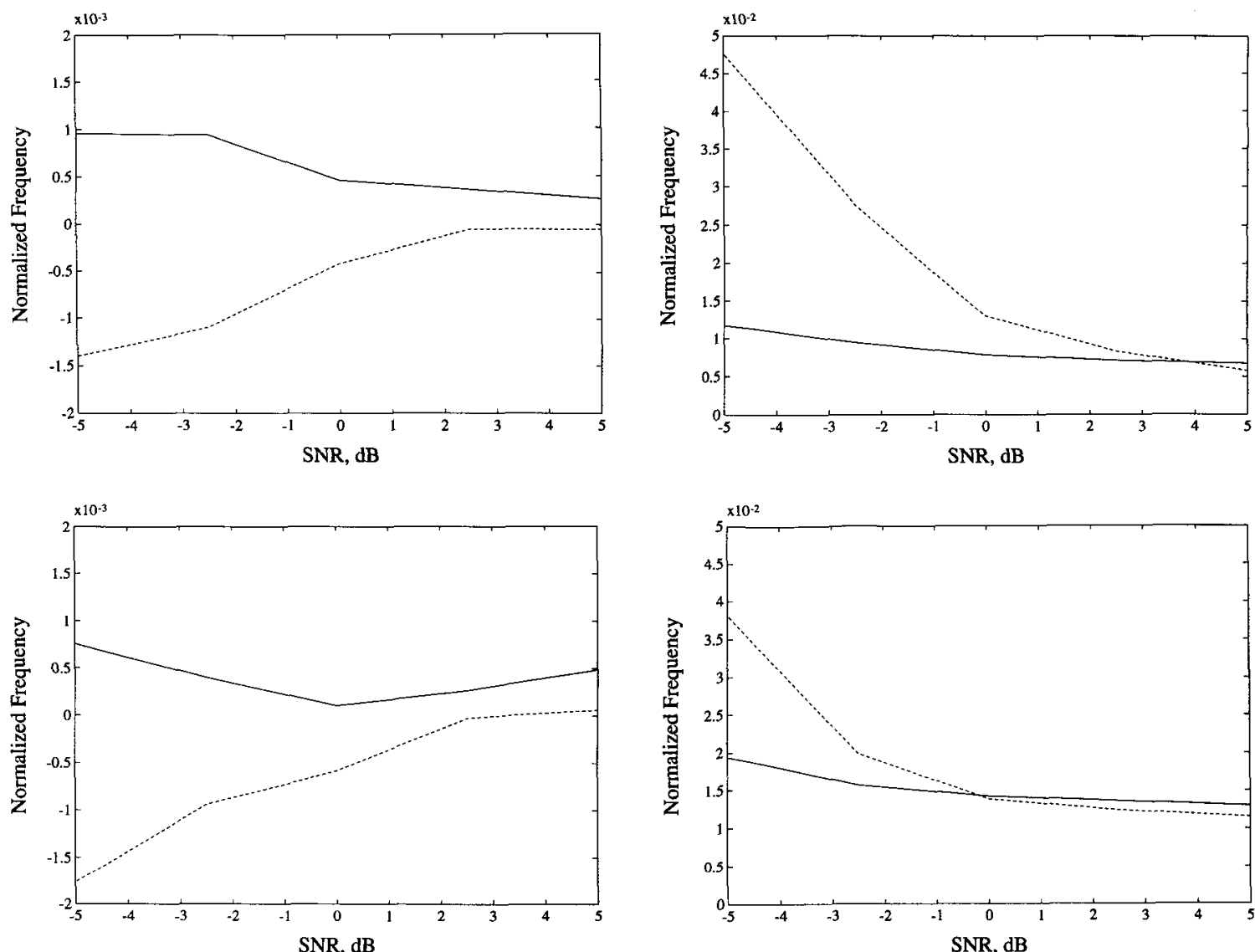

FIG. 5. Bias and standard deviation of ANF (solid line) and PPP (dotted line) frequency estimates vs SNR for two spectral widths $\sigma_{d l}\left(0.01 F_{s}\right.$ and $\left.0.03 F_{s}\right)$ : (a) bias for $\sigma_{d}=0.01 F_{s}$, (b) standard deviation for $\sigma_{d}=0.01 F_{s}$, (c) bias for $\sigma_{d}=0.03 F_{s}$, and (d) standard deviation for $\sigma_{d}=0.03 F_{s}$. The input signal frequency is $0.2 F_{s}$.

5\% with PPP-PO), convinced us to use PPP-PO as a reference.

\section{a. Stationary signals}

Stationary Doppler lidar signals were simulated according to Zrnić's model (1975). We remind the readers that this model operates in the frequency domain and consists in randomly modulating a Gaussianshaped power spectrum characterized by a central frequency and a standard deviation $\sigma_{d}$ (spectral width) plus a uniform level of white noise, and then of an inverse fast Fourier transform. From a practical point of view, the operations are performed on sampled power spectra made of $N$ frequency channels. Examples of simulated signal spectra are presented in Fig. 4 for various values of $\sigma_{d}$ and SNR with $N=4096$.

Biases and standard deviations were estimated from the frequency analysis both by PPP and ANF of 200 signals made of 4096 data samples each. For every signal, frequency profiles were obtained from PPP using a sliding window of 64 data samples (time duration of $128 T_{s}$ as the complex-valued signals are decimated by 2 ). The PPP estimate at time $k$ results in fact from the frequency analysis between points $k-32$ and $k+31$. As far as the ANF is concerned, the time resolution was tuned from $5\left(\lambda_{0}=0.8\right)$ to $20\left(\lambda_{0}=0.95\right)$ from the initial time onward, the final time resolution being reached at about time step 200. These figures were experimentally chosen as they appeared optimal to us. We point out that the time resolutions expressed here are anyway much lower than for PPP, and that the improvement of accuracy for the ANF that we will show hereafter cannot be, hence, attributed to a longer time resolution.

The PPP estimator worked on complex-valued signals exclusively, whereas the ANF has to be performed on real-valued signals. The comparison between both estimators was conducted as follows. A complex-valued signal with a frequency around $0.2 F_{s}$ was first simulated; when the PPP is concerned, this signal is decimated by two corresponding to a bandwidth $\left[-F_{s} / 4\right.$, 

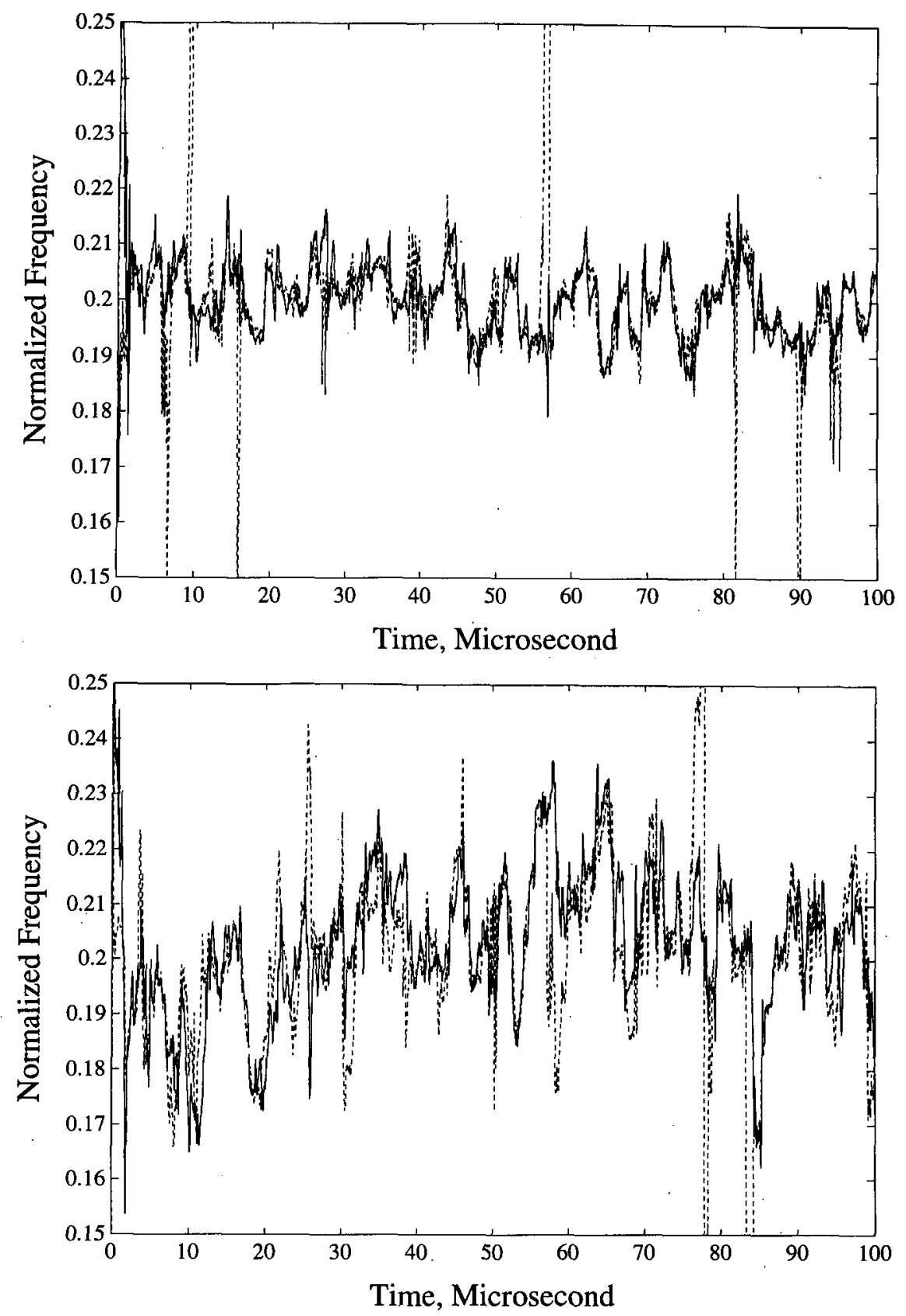

FIG. 6. ANF and PPP frequency estimates vs time in stationary conditions: (a) $\mathrm{SNR}=0 \mathrm{~dB}$ and $\sigma_{d}=0.01 F_{s}$, (b) $0 \mathrm{~dB}, 0.03 F_{s}$, (c) $-5 \mathrm{~dB}, 0.01 F_{s}$, and (d) $-5 \mathrm{~dB}, 0.03 F_{s}$. The input signal frequency is $0.2 F_{s}$.

$+F_{s} / 4 j$, the sampling frequency $F_{s} / 2$; when on the contrary the ANF was considered, the complex-valued signal was frequency shifted up from $F_{s} / 4$, and the real part was then extracted leading to the bandwidth $[0$, $\left.F_{s} / 2\right]$ and the sampling frequency $F_{s}$. Doing so enabled us to keep the same amount of information and the same SNR in both cases. In the figures, the frequency shift $+F_{s} / 4$ is removed from the ANF estimates for the sake of clarity.

Biases and standard deviations of both estimators are plotted versus SNR in Fig. 5 for two values $\sigma_{d}=0.01 F_{s}$ and $\sigma_{d}=0.03 F_{s}$. At low SNR (below $0 \mathrm{~dB}$ ), the standard deviation remains of the order of 0.015 (equivalent to a velocity of $3 \mathrm{~m} \mathrm{~s}^{-1}$ with $F_{s}=40 \mathrm{MHz}$ and a 

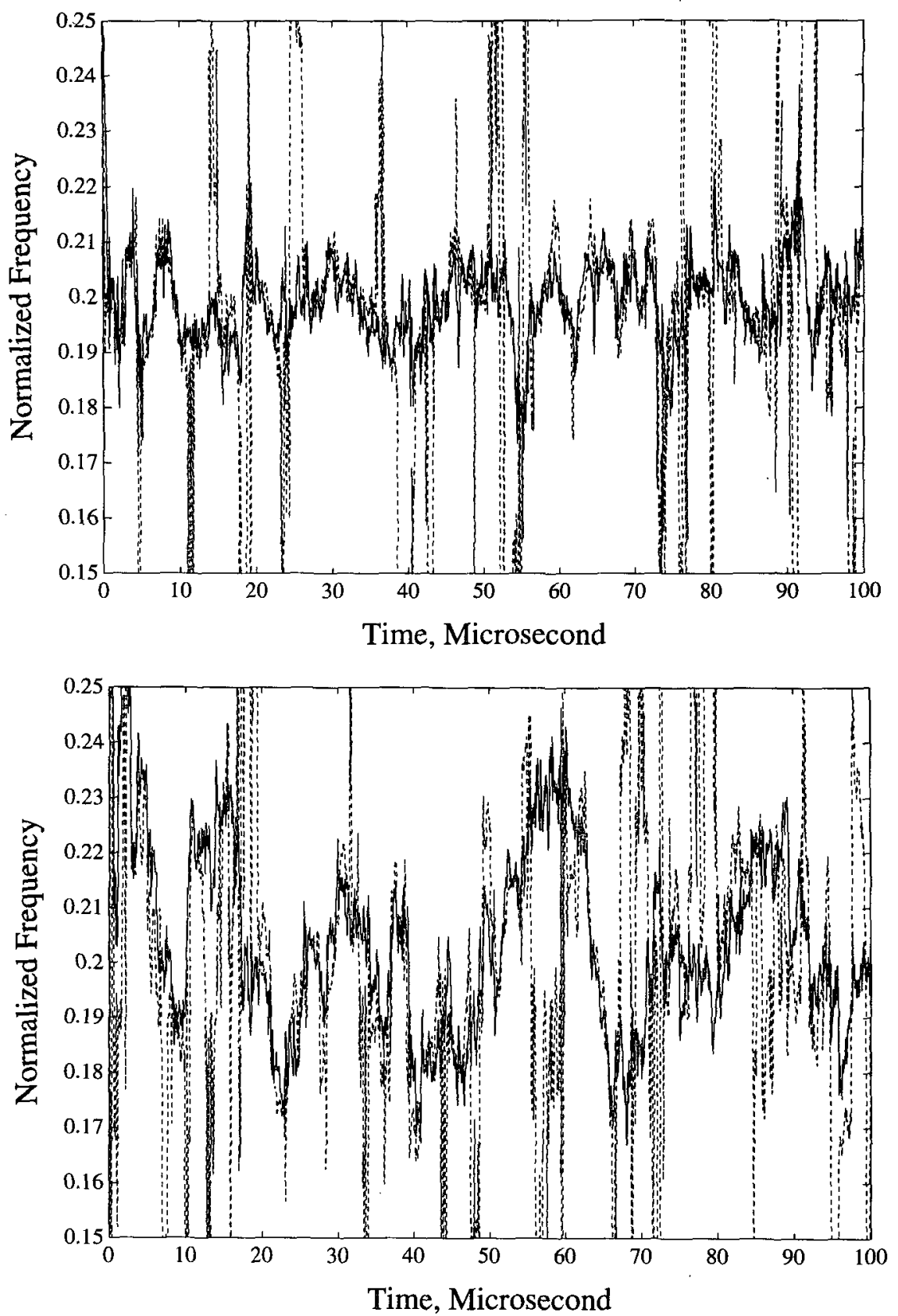

FIG. 6. (Continued)

laser wavelength of $10 \mu \mathrm{m})$ for the ANF while it can be as large as $0.045\left(9 \mathrm{~m} \mathrm{~s}^{-1}\right)$ for the PPP. The reason for such a discrepancy will be discussed later. At high SNR however, the accuracy of both estimators are saturating to a nonzero standard deviation, with the PPP slightly better than ANF. The fact that the estimates of both the PPP and ANF estimators are randomly fluctuating even though the SNR is very high, is due to the speckled random fluctuations of signal that make its frequency randomly fluctuating as well. These frequency fluctuations are indeed identified as measurement errors since the wind velocity is related to the mean signal frequency and not the instantaneous frequency. As far as the ANF is concerned, the errors at high SNR are larger because of the shorter time resolution, and the notch filter bandwidth, which, resulting from a trade-off between convergence and error, is not optimized to achieve a low variance only. Comparing 

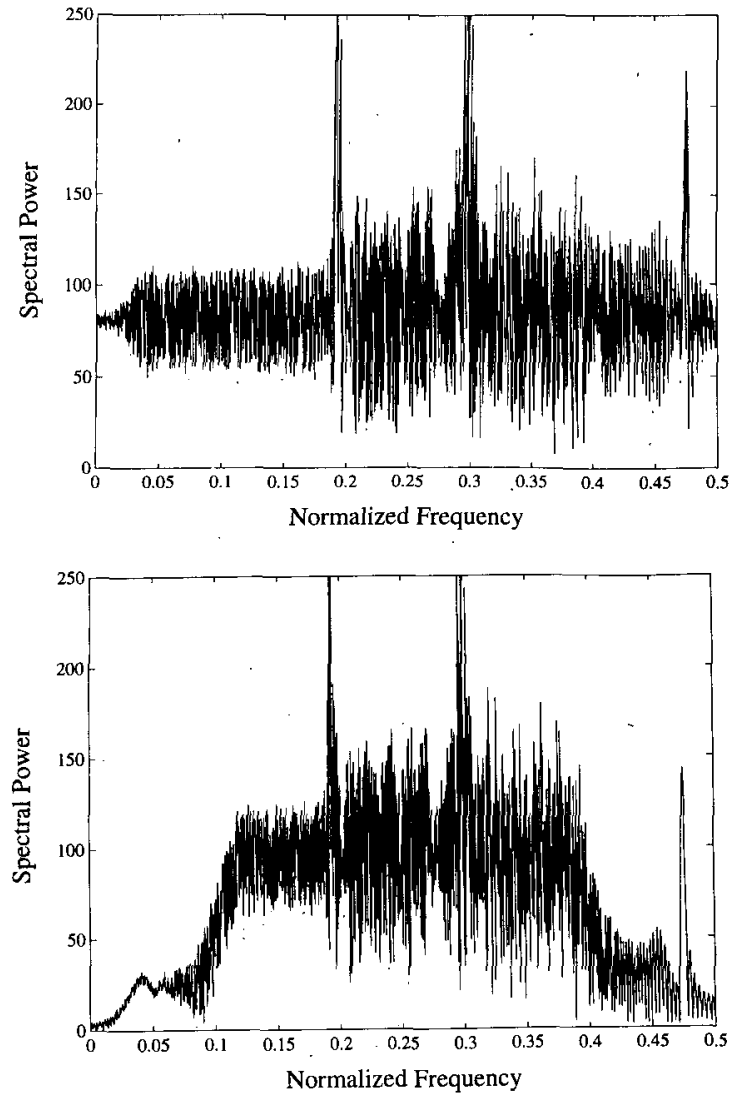

FiG. 7. Power spectra of signals with time-varying frequency: (a) white noise; (b) colored noise. The SNR is $-5 \mathrm{~dB}$ in both cases.

the PPP and ANF for both spectral widths, the improvement due to ANF is higher at $\sigma_{d}=0.01 F_{s}$ than at $\sigma_{d}$ $=0.03 F_{s}$. This result is not surprising since the ANF estimator is rather designed for narrowband signals.

The biases for both estimators are on the contrary rather similar and stay below $0.001 F_{s}$ even at low SNR.

Frequency profiles obtained with the two estimators are displayed in Fig. 6 where the SNR is equal to $0 \mathrm{~dB}$ or $-5 \mathrm{~dB}$. The PPP estimator sometimes displays outliers with an increasing probability when the SNR is decreasing and when the spectral width is increased. The ANF estimator does not display such outliers, because it is adaptive. The outliers are responsible for a great part of the standard deviation. The fact that they do appear with the PPP and not with the ANF (at least down to $-5 \mathrm{~dB}$ ) explains why the standard deviation displayed by PPP are larger at low SNR compared to ANF.

\section{b. Nonstationary signals with time-varying frequency}

Most of the studies on Doppler lidar signal processing were conducted on stationary signals using Zrnić's model. As we have pointed out in the previous para- graph, this is not however sufficient when analyzing the performance of an adaptive frequency estimator, for its accuracy results from two opposite criteria: the convergence time and the variance of the estimates. The convergence of the estimator, both at initialization and along the frequency analysis of a single lidar return, requires a study of the estimator's performance on nonstationary signals. We consider here signals with a time-varying frequency.

This study also considers the problem of a colored noise contaminating the signal. As far as the Doppler lidar is concerned, the need to work on a single return very likely brings such a colored noise when the signal is filtered for antialiasing purposes or noise power reduction (Fig. 7b). A colored noise may be detrimental to the accuracy of estimators. This is true for instance for the PP and PPP estimators that remove the noise on the assumption it is white. If this is not true, part of the noise remains within the signal, and the estimators are biased. The robustness of the adaptive notch filter with regards the color of noise if thus an important issue that needs to be studied further.

Nonstationary signals with white or colored noises are simulated in the time domain using a sine wave with a time-varying frequency. The frequency profile considered is shown in Fig. 8. The noise is added to the signal in the time domain. When a colored noise is considered, a spectral filter removing the spectral power outside the bandwidth $[0.1,0.4] F_{s}$ is used. In both cases, the resulting signal-to-noise ratio is $-5 \mathrm{~dB}$. An example of the resulting power spectra is shown in Fig. 7. A complex version of the signal to be processed with the PPP estimator is obtained by multiplying the original real-valued signal by $\exp \left(-2 i \pi \times 0.25 F_{s} t\right)$, and then by a filtering using a bandpass filter $[-0.25$, $0.25] F_{s}$. The PPP estimates displayed in Figs. 8-11 are shifted back around $0.25 F_{s}$ for comparison with the ANF.

In the case of a white noise (see Figs. 8 and 9), we notice that both PPP and ANF manage to track the signal frequency rather well, although some time delay does appear for both estimators when the signal frequency rapidly varies (between 65 and $75 \mu$ s for instance). It takes even a little bit longer for the ANF to recover the signal frequency.

When the noise is colored however (see Figs. 10 and 11 ), the accuracy is worse. As expected, this is particularly true for the PPP estimator, which confirms the sensitivity of PPP to colored noise. As shown in Fig. 11 which displays the average of 200 estimates, PPP is biased toward $0.25 F_{s}$ is the mean frequency of the colored noise. The ANF however better manages to track the signal frequency, with larger errors though when the signal frequency is close to the Nyquist's frequency. The ability of the ANF to tightly track the signal frequency could be improved by using a smaller $\lambda_{0}$ 


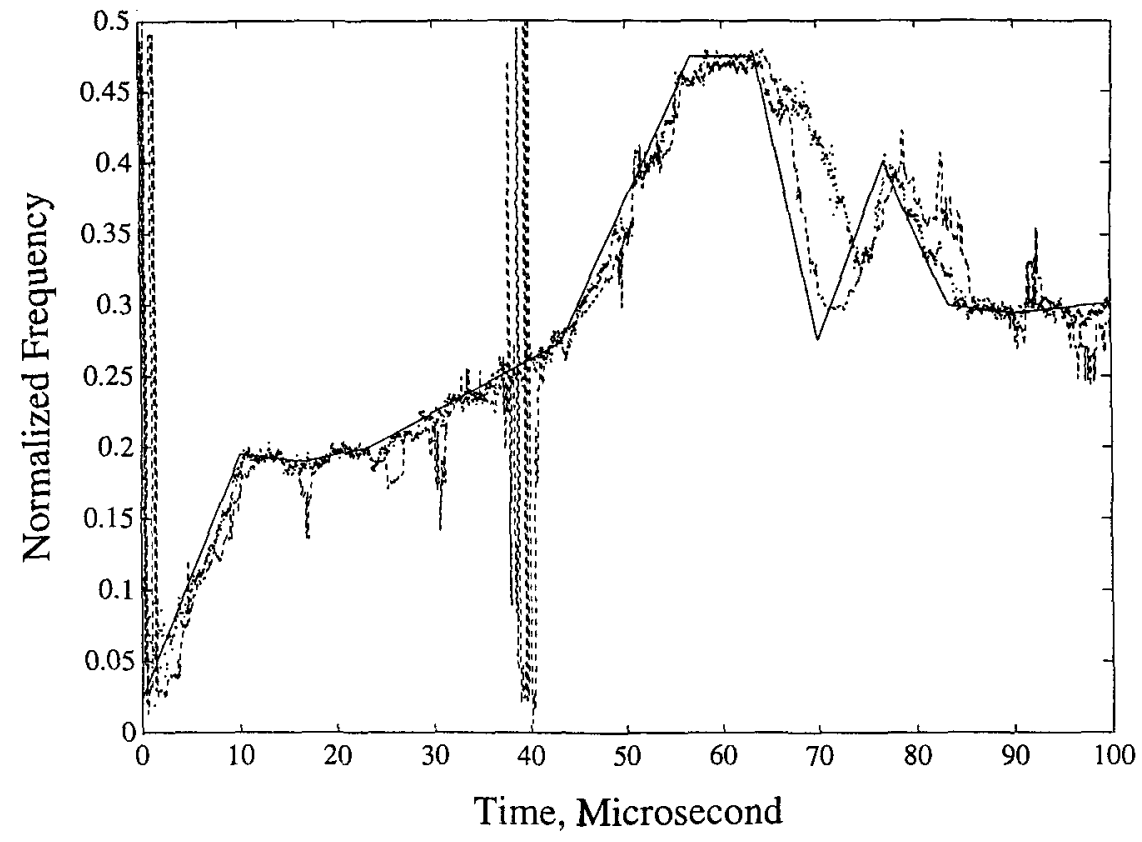

FlG. 8. Single-shot estimates of ANF (dotted line) and PPP (dashed line) vs time with white noise. $\mathrm{SNR}=-5 \mathrm{~dB}$. The reference frequency is displayed as solid line.

coefficient, but it would be detrimental to the variance of the estimates.

\section{Conclusions}

In this paper, we compared two different frequency estimators ANF and PPP for processing pulsed Doppler lidar signals. The ANF is proposed as an alternative to the well-known PPP processor. The ANF is based on the filtering of the lidar signal to separate the signal frequency from the noise contribution. An adaptive algorithm is used to retrieve the filter parameters at time $k$ knowing the input signal and filter output at time $k$

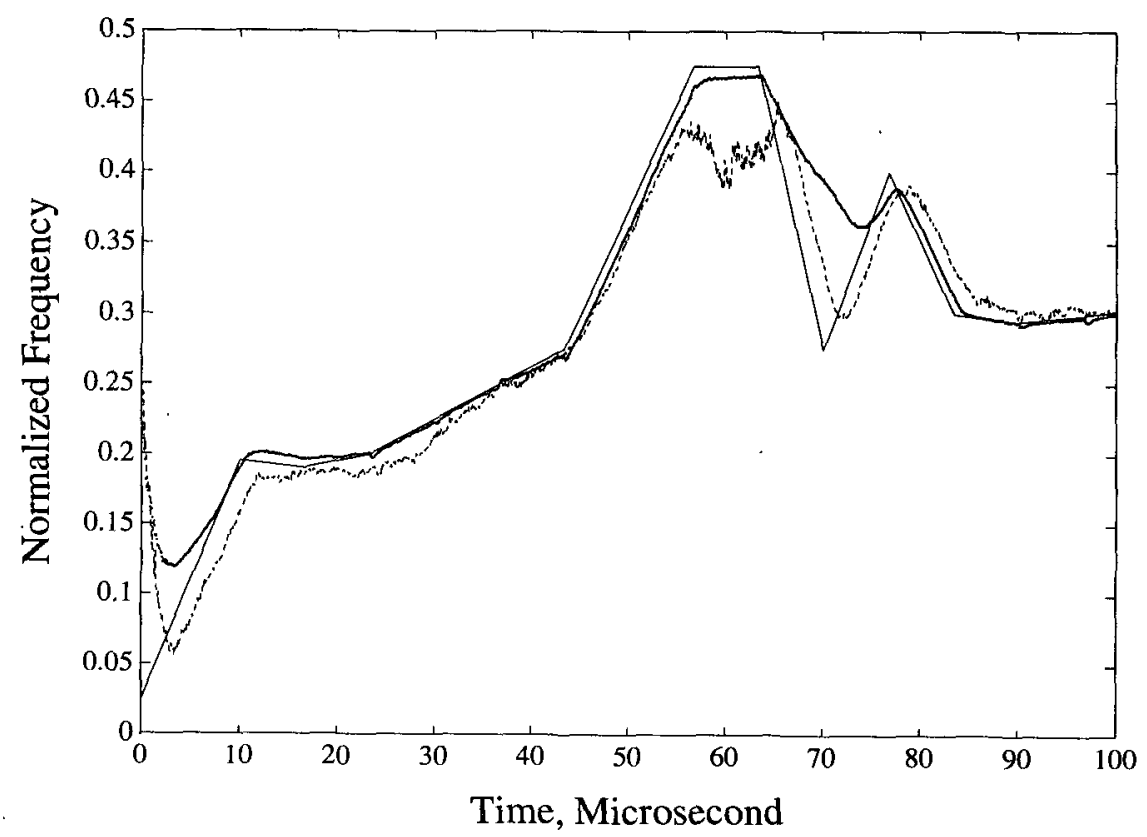

FIG. 9. Same as Fig. 8 except averaging over 200 estimates. 


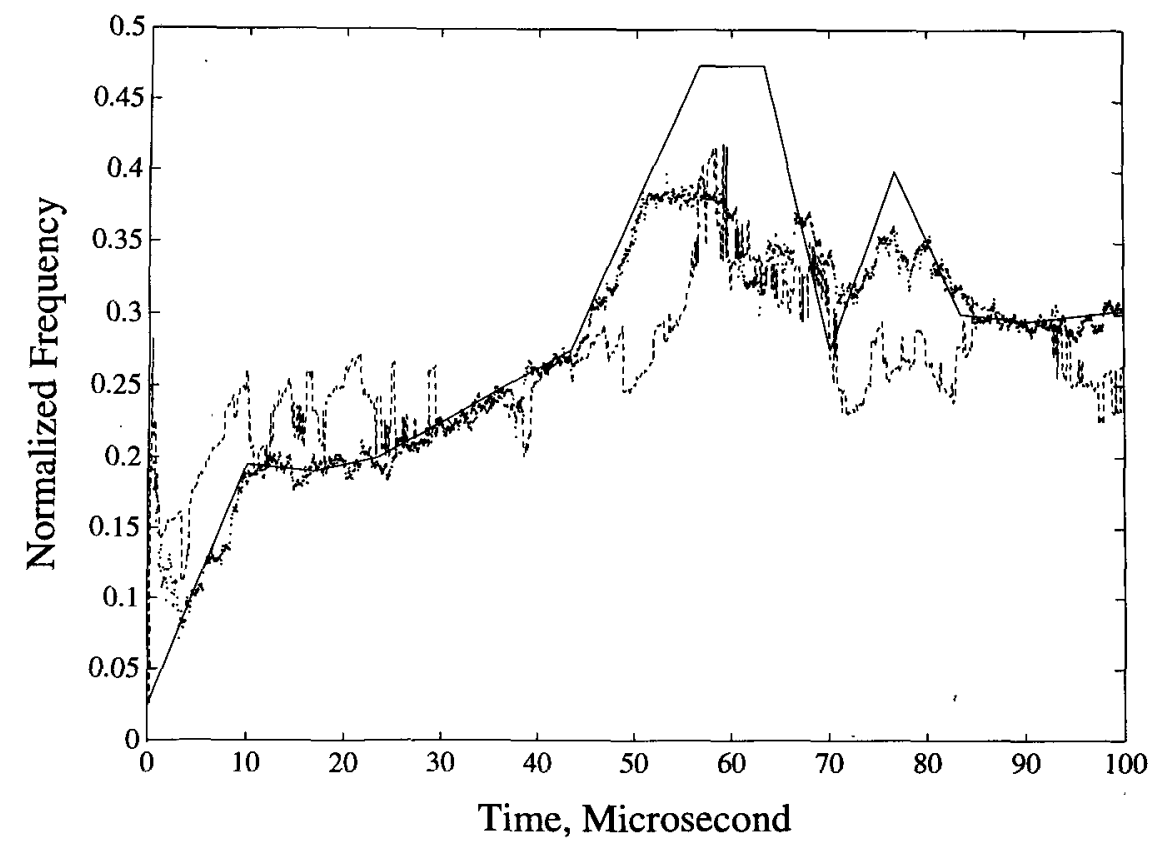

FIG. 10. Same as Fig. 8 but with colored noise.

$-i, i=[1, k]$. It is shown that the main advantage of this algorithm is the good performances at low signalto-noise ratio $(-5 \mathrm{~dB})$ due to its ability to avoid outliers. The standard deviation of the estimates once the convergence regime is reached is of the order of 0.01 0.02 (in normalized frequency unit) depending on the spectral width. This corresponds to a wind velocity uncertainty of the order of $2-4 \mathrm{~m} \mathrm{~s}^{-1}$ with $F_{s}=40 \mathrm{MHz}$ for a signal to noise ratio of $-5 \mathrm{~dB}$. The ANF also proved to perform better than PPP in tracking a timevarying frequency both in the case of a white or colored noises. The ANF should be however optimized to track

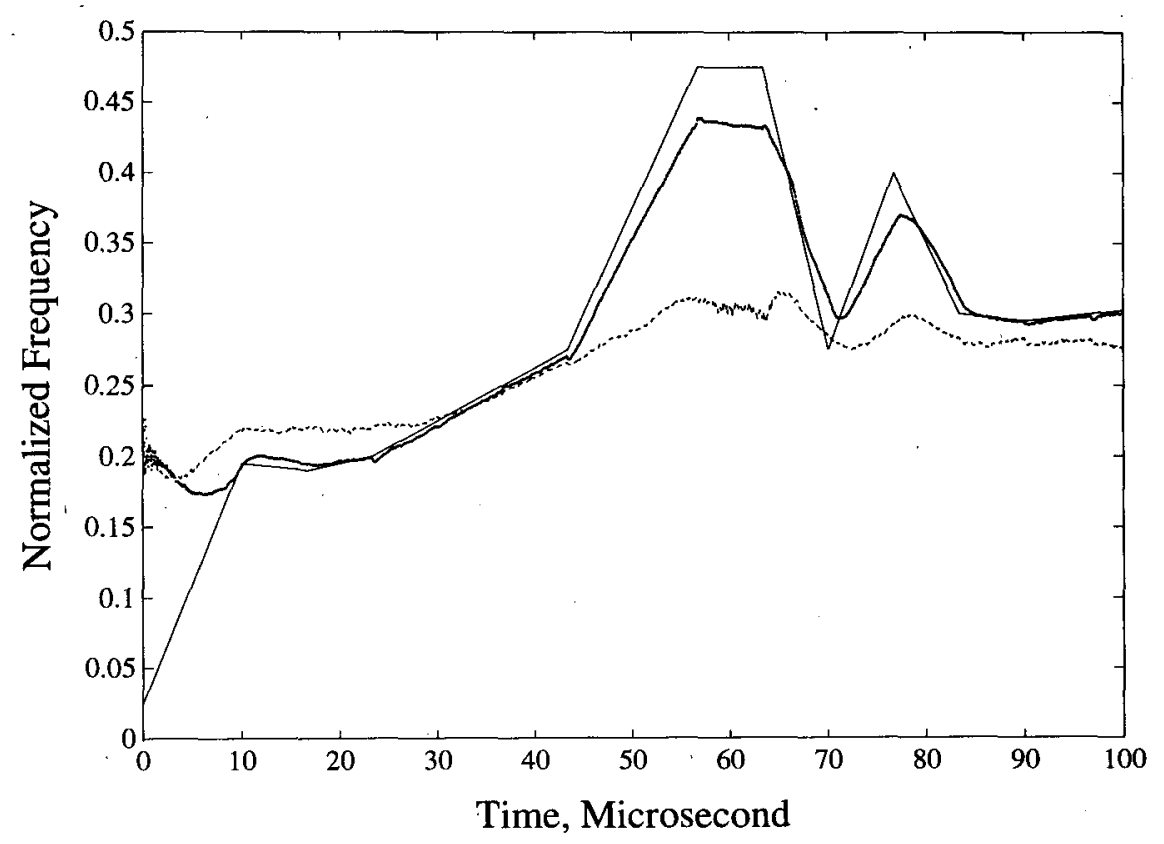

FIG. 11. Same as Fig. 9 but with colored noise. 
rapid wind variations at low SNR, although this would bring a larger variance of the frequency estimates. Since the numerical implementation of the ANF requires more computing resources than the simple PPP processor, fast-floating point processor are now available to design sophisticated processing schemes.

APPENDIX A

\section{Optimal Predictor}

The purpose of the appendix is to show that the optimal prediction for the notch filter input signal $x(k)$ is reached when the prediction error is equal to the noise term (i.e., to the output of the notch filter). Let us define $p(k)$ as the optimal predictor of $x(k)$; it depends of $x(k)$ and $\tilde{n}(k)$ at time $k-i, i=[1, k]$. The prediction is optimal when the mean quadratic error $J_{k}$ $=E\left\{[x(k)-p(k)]^{2}\right\}$ is minimum. Using (8):

$$
\begin{aligned}
p(k)=-\hat{a}_{1}(k) x(k-1)-x(k-2) & \\
& +\hat{a}_{1}(k) r \tilde{n}(k-1)+r^{2} \tilde{n}(k-2)
\end{aligned}
$$

one obtains

$$
\begin{aligned}
J_{k} & =E\left\{\left[-\hat{a}_{1}(k) x(k-1)-x(k-2)+\hat{a}_{1}(k) r \tilde{n}(k-1)+r^{2} \tilde{n}(k-2)-p(k)+\tilde{n}(k)\right]^{2}\right\} \\
& =E\left\{\left[-\hat{a}_{1}(k) x(k-1)-x(k-2)+\hat{a}_{1}(k) r \tilde{n}(k-1)+r^{2} \tilde{n}(k-2)-p(k)\right]^{2}\right\}+E\left[\tilde{n}^{2}(k)\right] \\
& \quad+2 E\left\{\left[-\hat{a}_{1}(k) x(k-1)-x(k-2)+\hat{a}_{1}(k) r \tilde{n}(k-1)+r^{2} \tilde{n}(k-2)-p(k)\right] \tilde{n}(k)\right\} .
\end{aligned}
$$

Since $\tilde{n}(k)$ is a zero-mean white noise, $J_{k}$ can be written as

$$
\begin{aligned}
J_{k}=E & \left\{\left[-\hat{a}_{1}(k) x(k-1)\right.\right. \\
- & x(k-2)+\hat{a}_{1}(k) r \tilde{n}(k-1) \\
& \left.\left.+r^{2} \tilde{n}(k-2)-p(k)\right]^{2}\right\}+E\left[\tilde{n}^{2}(k)\right] .
\end{aligned}
$$

The minimization of (A2) requires the minimization of the first rhs term since $E\left[\tilde{n}^{2}(k)\right]$ is not predictible. We thus have

$$
\begin{aligned}
p(k)=-\hat{a}_{1}(k) x(k-1)-x(k-2) & \\
& +\hat{a}_{1}(k) r \tilde{n}(k-1)+r^{2} \tilde{n}(k-2) .
\end{aligned}
$$

\section{APPENDIX B}

\section{Minimization of the Mean Quadratic Error $\hat{J}_{k}$}

At time $k$, the optimal prediction is obtained by minimizing the mean quadratic error $\hat{J}_{k}$ defined in (13):

$$
\hat{J}_{k}=\frac{1}{k} \sum_{i=1}^{k} \lambda_{k}(i)\left[x(i)-\boldsymbol{\theta}^{\mathrm{T}}(k) \phi(i)\right]^{2} .
$$

This is achieved by equating to zero $\hat{J}_{k}$ derivatives with respect to $\theta(k)$ :

$$
\begin{aligned}
\frac{d \hat{J}_{k}}{d \boldsymbol{\theta}(k)}=-\frac{2}{k} \sum_{i=1}^{k} \lambda_{k}(i) & {[x(i)} \\
& \left.-\boldsymbol{\theta}^{\mathrm{T}}(k) \boldsymbol{\phi}(i)\right] \Psi^{\mathrm{T}}(i)=0,
\end{aligned}
$$

where $\boldsymbol{\Psi}^{\mathrm{T}}(i)$ is defined by

$$
\Psi^{\mathrm{T}}(i)=-\frac{d\left[x(i)-\boldsymbol{\theta}^{\mathrm{T}}(k) \boldsymbol{\phi}(i)\right]}{d \boldsymbol{\theta}(k)} .
$$

It is shown by Nehoraï (1985) and M'Sirdi (1987) that
$\Psi^{\mathrm{T}}(i)$ may be calculated recursively from the vectors $\phi(i)$ :

$$
\boldsymbol{\Psi}^{\mathrm{T}}(i)+\hat{a}_{1}(k) r \boldsymbol{\Psi}^{\mathrm{T}}(i-1)+r^{2} \boldsymbol{\Psi}^{\mathrm{T}}(i-2)=\boldsymbol{\phi}(i) .
$$

The solution of (B1) is then equal to

$$
\begin{aligned}
\boldsymbol{\theta}(k)= & {\left[\sum_{i=1}^{k} \lambda_{k}(i) \Psi(i) \boldsymbol{\phi}^{\mathrm{T}}(i)\right]^{-1} \sum_{i=1}^{k} \lambda_{k}(i) x(i) \Psi(i) } \\
= & \mathbf{F}(k) \sum_{i=1}^{k} \lambda_{k}(i) x(i) \Psi(i) \\
= & \mathbf{F}(k) \sum_{i=1}^{k-1} \lambda_{k}(i) x(i) \Psi(i) \\
& +\mathbf{F}(k) x(k) \Psi(k),
\end{aligned}
$$

where

$$
\begin{gathered}
\mathbf{F}(k)=\left[\sum_{i=1}^{k} \lambda_{k}(i) \Psi(i) \boldsymbol{\phi}^{\mathrm{T}}(i)\right]^{-1} \\
\mathbf{F}^{-1}(k)=\lambda_{0} \mathbf{F}^{-1}(k-1)+\Psi(k) \boldsymbol{\phi}^{\mathrm{T}}(k) .
\end{gathered}
$$

Considering the matrix inversion lemma (Ljung and Soderström 1983), $\mathbf{F}(k)$ can be calculated recursively from $\Psi(k)$ and $x(k)$ :

$$
\begin{aligned}
\mathbf{F}(k)= & \frac{1}{\lambda_{0}}[F(k-1) \\
& \left.-\frac{F(k-1) \Psi(k) \phi^{\mathrm{T}}(k) F(k-1)}{\lambda_{0}+\phi^{\mathrm{T}}(k) F(k-1) \Psi(k)}\right] .
\end{aligned}
$$

Using (B4) and (B6), the filter parameters $\theta(k)$ can also be recursively calculated: 


$$
\boldsymbol{\theta}(k)=\boldsymbol{\theta}(k-1)+\mathbf{F}(k-1) \Psi(k) \epsilon(k),
$$

where

$$
\epsilon(k)=\frac{x(k)-\phi^{\mathrm{T}}(k) \boldsymbol{\theta}(k-1)}{\lambda_{0}+\boldsymbol{\phi}^{\mathrm{T}}(k) \mathbf{F}(k-1) \Psi(k)}
$$

\section{REFERENCES}

Ancellet, G., R. T. Menzies, and W. B. Grant, 1989: Atmospheric velocity spectral width measurements using the statistical distribution of pulsed $\mathrm{CO}_{2}$ lidar return signal intensities. J. Atmos. Oceanic Technol., 6, 50-58.

Bilbro, J. W., G. Fichlt, D. Fitzjarrald, M. Krause, and R. Lee, 1984: Airborne Doppler lidar wind field measurements. Bull. Amer. Meteor. Soc., 65, 348-359.

_- C. DiMarzio, D. Fitzjarrald, S. Johnson, and W. Jones, 1986: Airborne Doppler lidar measurements. Appl. Opt., 25, 39523960.

Burg, J. P., 1967: Maximum entropy spectral analysis. Proc. 37th Meeting Society of Exploration Geophysicists, Oklahoma City, OK.

Dolfi-Bouteyre, A., C. Loth, G. Ancellet, and P. H. Flamant, 1988: A coherent pulsed $\mathrm{CO}_{2}$ lidar system for backscatter measurement. 14th International Laser Radar Conf., San Candido, Italy, Consiglio Nazionale delle Ricerche, 444-447.

Doviak, R. J., and D. S. Zrnić, 1984: Doppler Radar and Weather Observations. Academic Press, 458 pp.

Glover, J. R., 1977: Adaptive noise cancelling applied to sinusoidal interference. IEEE Trans. on Acoustic, Speech and Signal Processing, 27, 484-491.

Hardesty, R. M., T. R. Lawrence, R. A. Richter, M. J. Post, F. F. Hall Jr., and R. M. Huffaker, 1983: Ground based coherent lidar measurement of tropospheric and stratospheric parameters. Proc. SPIE, 85-91.

Lee, R. W., and K. A. Lee, 1980: A poly pulse pair signal processor for coherent Doppler lidar. Topical Meeting on Coherent Laser Radar for Atmospheric Sensing, Aspen, CO, OSA 1-4.
Ljung, L., and T. Soderström, 1983: Theory and Practice of Recursive Identification. MIT Press, $525 \mathrm{pp}$.

Mahapatra, P. R., and D. S. Zrnic, 1983: Practical algorithms for mean velocity estimation in pulse Doppler weather radars using a small number of samples. IEEE Trans. Geosci. Remote Sens., GE-21, 491-501.

Menzies, R. T., and R. M. Hardesty, 1989: Coherent Doppler lidar for measurements of wind fields. Proc. IEEE, 77, 449-462.

Miller, K. S., and M. M. Rochwarger, 1972: A covariance approach to spectral moment estimation. IEEE Trans. Inform. Theory, IT$18,588-596$.

M'Sirdi, N. K., 1987: Modélisation paramétrique adaptative et applications à l'analyse spectrale. Doctoral dissertation, University of Grenoble, 268 .pp.

Nehorai, N., 1985: A minimal parameter adaptive notch filter with constrained poles and zeros. IEEE Trans. Acoustic, Speech Signal Processing, 33, 983-996.

Neiman, P. J., M. A. Shapiro, R. M. Hardesty, B. B. Stankov, R. T. Lawrence, R. J. Zamora, and T. Hampel, 1988: The pulsed coherent Doppler lidar: observations of frontal structure and the planetary boundary layer. Mon. Wea. Rev., 116, 1671-1681.

Palmer, R. D., J. R. Cruz, and D. S. Zrnić, 1991: Enhanced autoregressive moving average spectral estimation applied to the measurement of Doppler spectral width. IEEE Trans. Geosci. Remote Sens., GE-29, 358-368.

Post, M. J., and W. D. Neff, 1986: Doppler lidar measurement of winds in a narrow mountain valley. Bull. Amer. Meteor. Soc., 67, 274-281.

Rabiner, R. L., and B. Gold, 1975: Theory and Application of Digital Signal Processing. Prentice-Hall, 772 pp.

Rothermel, J., C. Kessinger, and D. L. Davis, 1985: Dual-Doppler lidar measurement of winds in the JAWS experiment. J. Atmos. Oceanic Technol., 2, 138-147.

Widrow, B., and S. D. Stearn, 1985: Adaptive Signal Processing. Prentice-Hall, 472 pp.

Zrnić, D. S., 1975: Simulation of weatherlike Doppler spectra and signals. J. Appl. Meteor., 14, 619-620.

, 1979: Estimation of spectral moments for weather echoes. IEEE Trans. Geosci. Electron., GE-17, 113-128. 